\title{
Cognitive and adaptive deficits in young children with Duchenne muscular dystrophy (DMD)
}

\author{
SHANA E. CYRULNIK, ${ }^{1,2}$ ROBERT J. FEE, ${ }^{2}$ ABIGAIL BATCHELDER, ${ }^{2}$ JACQUELINE KIEFEL, ${ }^{3}$ \\ EDWARD GOLDSTEIN, ${ }^{4}$ AND VERONICA J. HINTON ${ }^{2,5}$ \\ ${ }^{1}$ The Graduate Center of the City University of New York, Manhattan, New York \\ ${ }^{2}$ Gertrude H. Sergievsky Center, Columbia University, Manhattan, New York \\ ${ }^{3}$ Department of Neuropsychology, Children's Healthcare of Atlanta at Scottish Rite, Atlanta, Georgia \\ ${ }^{4}$ Department of Neurology, Children's Healthcare of Atlanta at Scottish Rite, Atlanta, Georgia \\ ${ }^{5}$ College of Physicians \& Surgeons, Columbia University, Manhattan, New York
}

(Received March 17, 2006; Final Revision June 2, 2008; Accepted June 3, 2008)

\begin{abstract}
The goal of the current investigation was to examine adaptive behavior and cognitive skills in young children with Duchenne muscular dystrophy (DMD), a genetic disorder that causes progressive muscular weakness and concomitant cognitive deficits. Previous studies have documented specific language deficits in older children with DMD, but there are limited data on younger children. Twenty children with DMD who were between 3 and 6 years old and 20 unaffected family control children were recruited. Parents completed questionnaires relating to development and adaptive functioning, while children completed neuropsychological testing. Results of paired $t$ tests indicate that children with DMD are rated as delayed relative to familial controls on measures of adaptive functioning, as assessed by the Vineland Adaptive Behavior Scales. Furthermore, children with DMD exhibit impairments on multiple measures of cognition, including measures of receptive language, expressive language, visuo-spatial skills, fine-motor skills, attention, and memory skills. Across all domains examined, the young children with DMD performed more poorly than their familial controls. These deficits appear to be more generalized than those reported in older children with this disorder. Dystrophin, a missing protein product, is hypothesized to be responsible for these cognitive and behavioral impairments. (JINS, 2008, 14, 853-861.)
\end{abstract}

Keywords: Development, Language, Preschool, Dystrophin, Genetic, Neuroscience

\section{INTRODUCTION}

Research in the field of developmental neuropsychology typically focuses on the study of children with various developmental and behavioral disorders. These disorders are usually characterized by well-defined clusters of symptoms, often with an unknown etiology. The current investigation focused on the reverse scenario: a disorder for which the genetic etiology is known, but the developmental symptoms are not nearly as well-documented. Delineating the symptoms associated with a disorder of known genetic etiology offers developmental neuropsychologists the tantalizing possibility that one can reduce neuropsychological differences to the level of the gene.

Correspondence and reprint requests to: Shana E. Cyrulnik, 530 West 236th Street, \#1K, Bronx, New York 10463. E-mail: shanacyrulnik@ pobox.com
The current investigation focused on Duchenne muscular dystrophy (DMD), a disorder of known genetic etiology, which is caused by a mutation on the $\mathrm{X}$ chromosome. It is the second most common single gene disorder, occurring in 1/3500 live male births (Emery \& Muntoni, 2003). Male children affected with this disease suffer from progressive, and ultimately fatal, muscular weakness. While known primarily for its devastating motor effects, DMD is also associated with cognitive deficits. These cognitive deficits have been studied in older children and adolescents with DMD. However, there are little data examining the development of cognitive skills in young male children with this disorder. The current investigation focused directly on the early development of cognitive skills in young boys with DMD.

The cognitive deficits documented in older children and adolescents with DMD appear to be mainly circumscribed to verbal skills. Across studies, verbal intelligence scores are significantly lower than performance intelligence scores (Cot- 
ton et al., 2001). Other areas of cognitive functioning, such as visuo-spatial skills, long-term memory, and abstract reasoning skills, do not appear to be affected (Cotton et al., 1998; Hinton et al., 2001; Karagan et al., 1980; Wicksell et al., 2004).

While there is a general consensus among investigators that verbal skills are preferentially affected in older children and adolescents with DMD, the nature of this deficit remains unclear. We have previously characterized this deficit as one of limited verbal span, based upon evidence which indicates that these children have considerable difficulty with immediate repetition of verbal material, and in particular, when the verbal information increases in length and complexity. When asked to recall numbers, sentences, or stories, for example, children with DMD consistently perform more poorly than their matched controls (Anderson et al., 1988; Billard et al., 1992, 1998; Dorman et al., 1988; Hinton et al., 2000, 2001, 2004; Ogasawara, 1989; Whelan, 1987; Wicksell et al., 2004). These findings appear across all intellectual levels, regardless of cognitive functioning (Hinton et al., 2000), and are not due to more general impairments in language and memory (Hinton et al., 2007). Whether limited verbal span reflects weaknesses in attentional or language skills is unclear, but most likely both skills contribute. We have further hypothesized that limited verbal span may be the core deficit in DMD. As deficits in verbal span have been linked to impairments in the acquisition of phonological knowledge and single-word vocabulary (Adams \& Gathercole, 2000; Gathercole et al., 1997), this core deficit may also help to explain impairments seen in phonological awareness, reading, and writing found in children with DMD (Billard et al., 1992; Dorman et al., 1988; Hendriksen \& Vles, 2006; Hinton et al., 2001, 2004; Leibowitz \& Dubowitz, 1981; Worden \& Vignos, 1962).

In comparison to the data regarding verbal deficits in older children and adolescents with DMD, there is relatively little information available regarding early development of language in DMD. Several case studies have described young children (e.g., ages 3-6 years) who came to clinical attention for significant language and behavioral problems and were subsequently diagnosed with DMD (Essex \& Roper, 2001; Kaplan et al., 1986; Mohamed et al., 2000). One of the few published data sets on young children with DMD (under the age of 72 months) is by Smith and colleagues (1990). The sample was comprised of 33 British children with DMD and normal, gender- and agematched controls. Compared with controls, children with DMD exhibited generalized developmental delay, with severe deficits in motor and language skills. Smith et al. (1990) also reported the presence of behavioral problems in the DMD group only. These data are somewhat at odds with the specificity of findings in older children. They suggest that in younger children with DMD, there may be early generalized developmental delays with concomitant behavioral problems, or that Smith's sample is very different from other groups in phenotypic characteristics as well as age. Given the paucity of data among young children with DMD, there is a strong need to replicate the findings and to help better characterize the cognitive and behavioral profiles of young children diagnosed with DMD. Moreover, in young children, delayed cognitive skills likely impact upon adaptive behavior and an evaluation of daily life skills is warranted.

The purpose of the current study was to explore adaptive behavior and cognitive skills of young children with DMD and to replicate the findings of early, generalized delay as documented by Smith et al. (1990). Adaptive behavior and cognitive skills were individually assessed in 3- to 6-yearold children with DMD and unaffected family controls to determine whether selective or generalized deficits are associated with a diagnosis of DMD. The following three hypotheses were evaluated: (1) Parents will rate young children with DMD as having poorer adaptive behavior skills than unaffected familial controls. (2) Children with DMD will perform more poorly on tests of cognitive functioning than their familial controls. (3) Young children with DMD will exhibit more generalized deficits than those reported in older children with DMD.

\section{METHODS}

\section{Participants}

Twenty young boys with muscular dystrophy who were between the ages of 3 and 6 were recruited (mean age $=$ 4.90; $S D=1.12$ ). Inclusion criteria included diagnosis of muscular dystrophy, good general health, ability to complete all test measures, English as the primary language, and willingness to participate. All probands were ambulatory at the time of assessment.

Familial controls were recruited for each boy with DMD. Inclusion criteria consisted of proximity in age to the affected child (i.e., within 4 years), good general health, ability to complete all test measures, English as the primary language, and willingness to participate. Twenty subjects were recruited, consisting predominantly of siblings $(n=17)$. When more than one sibling was available, preference was given first to male gender and then to closeness in age. When no sibling was available, cousins $(n=3)$ were recruited; these cousins were similar in age, gender and socio-economic status to the child with DMD. Controls ranged in age from 3 to 9 , with a mean age of 5.10 years ( $S D=1.74)$. Among the controls, 9 were older $(6$ males and 3 females) and 9 ( 4 males and 5 females) were younger. Two controls were fraternal twins ( 1 male, 1 female). Age was comparable between the proband and control groups $(t=.43$, not significant [n.s.]).

The majority of probands and controls had not yet entered first grade; they were in preschool, prekindergarten, or kindergarten. Education level was comparable between the two groups $\left(\chi^{2}=9.94\right.$, n.s. $)$. The majority of young children with DMD were receiving therapeutic services; physical (86\%), occupational (77\%), and speech (59\%). In contrast, a smaller percentage of controls were receiving speech therapy services (13\%). 
Racial composition of the sample was predominantly Caucasian (92\%), with an additional $6 \%$ reported to be Hispanic; $2 \%$ did not categorize their racial affiliation. Responses to questions about socio-economic information indicated that the sample was composed of well-educated families. Most mothers had completed a Bachelor's degree, and the average family income was between $\$ 75,000$ and $\$ 99,999$.

\section{Procedure}

Participants for this study were recruited through the Muscular Dystrophy Association (MDA) clinics of Columbia Presbyterian Hospital, New York, and Children's Healthcare of Atlanta at Scottish Rite, Atlanta, Georgia. Additionally, newsletters with a description of the study were sent to Parent Project Muscular Dystrophy (PPMD), regional MDA clinics, and parent support groups. Interested individuals returned the response form directly to the investigator. This study was approved by the Columbia University and New York Presbyterian Hospital Institutional Review Board, by the Queens College of the City University of New York Institutional Review Board, and by the Children's Healthcare of Atlanta at Scottish Rite Children's Medical Center Institutional Review Board.

Written informed consent was provided by all parents before their children's participation. Children who were capable of doing so gave verbal assent before their participation. Parents completed the questionnaires while their children were being tested. Testing was done in English. The entire battery of tests took approximately $4 \mathrm{hr}$ to administer, and testing was generally divided into two separate sessions of $2 \mathrm{hr}$ each, so as not to over-burden the children.

Although testers were not blind to the child's diagnosis, test administration was standardized for both probands and controls. All tests were scored twice to ensure reliability of the data; discrepancies were resolved by consensus. To ensure that there was no evidence of hearing loss, a brief hearing screen was attempted with all research subjects. Approximately half the sample objected to the placement of the headphones on their head, or did not comply with the instructions. In those cases, medical records were reviewed and parents were interviewed to confirm that there was no suspicion of any auditory impairment.

\section{Measures: Parent battery}

Parents completed a developmental history form that queried about general health characteristics, schooling, and demographic information. In addition, parents completed the Vineland Adaptive Behavior Scales (Sparrow et al., 1984), a semi-structured interview designed to measure adaptive behavior. The questionnaire generates scores in four different areas of functioning, including Communication, Daily Living Skills, Socialization and Motor Skills, as well as a summary Adaptive Behavior Composite score, as an estimate of everyday living skills.

\section{Measures: Children's battery}

The following battery was composed of tests which have a minimal amount of motor demand, with the exception of tests specifically designed to measure fine-motor skills. The measures included tests of specific cognitive skills and select subtests from neuropsychological test batteries. Tests were grouped according to their presumed primary neuropsychological function. Six groups of measures were studied: (1) Receptive language skills, (2) expressive language skills, (3) visuo-spatial skills, (4) fine-motor skills, (5) attention skills, and (6) memory skills.

\section{Verbal skills: Receptive and expressive language skills}

The receptive composite score consisted of the Peabody Picture Vocabulary Test (PPVT-III) (Dunn \& Dunn, 1997), and three subtests from the Clinical Evaluation of Language Fundamentals-Preschool Version (CELF-P) (Wiig et al., 1992), which form the Receptive Language score (Linguistic Concepts, Sentence Structure, and Basic Concepts). The receptive composite score was derived by averaging the age-standardized scores of the tests included in this domain.

The expressive composite score consisted of the Expressive Vocabulary Test (EVT) (Williams, 1997), and three subtests from the CELF-P which form the Expressive Language score (Recalling Sentences, Formulating Labels, and Word Structure). This composite score was derived by averaging the age-standardized scores of the tests included in this domain.

\section{Visual-spatial skills}

The visual-spatial composite score consisted of the Matching subtest from the Wide Range Assessment of Visual Motor Abilities (WRAVMA) (Adams \& Sheslow, 1995), and Picture Completion from the Wechsler Preschool and Primary Scale of Intelligence-Revised (WPPSI-R) (Wechsler, 1989) or the Wechsler Intelligence Scale for Children-Third Edition (WISC-III) (Wechsler, 1991). The visual-spatial composite score was derived by averaging the age-standardized scores of the tests included in this domain.

\section{Fine-motor skills}

Fine-motor skills were assessed using the Pegboard subtest of the WRAVMA.

\section{Attention skills}

Attention skills were evaluated using the Visual Attention subtest of the NEPSY: A Developmental Neuropsychological Assessment (Korkman et al., 1997). Scaled scores derived from the NEPSY were transformed into standard scores. 


\section{Memory skills}

Memory skills were evaluated using the Narrative Memory subtest of the NEPSY: A Developmental Neuropsychological Assessment. Scaled scores derived from the NEPSY were transformed into standard scores.

\section{Analyses}

Paired $t$ tests were used to assess group differences in both adaptive behavior and cognitive functioning. To determine whether parents rate young children with DMD as having poorer adaptive behavior skills than their unaffected controls, the following four subtests of the Vineland were used as dependent variables: communication, socialization, daily living skills, and motor skills. The null hypothesis that children in both groups would have similar adaptive functioning in all domains was tested.

To determine whether children with DMD perform more poorly on tests of cognitive function than their controls, the following six composite scores served as the dependent variables: (1) Receptive language skills, (2) expressive language skills, (3) visuo-spatial skills, (4) fine-motor skills, (5) attention skills, and (6) memory skills. The null hypothesis that the two groups would perform similarly across all test measures was tested.

To examine whether gender or age of control had any modifying effects on outcome, multiple exploratory analyses of variance were run on the group differences scores of each composite cognitive variable using either gender of control or age of control (categorized as older, same, or younger) as the between-group factor. Exploratory analyses confirmed that neither gender nor age of control participant contributed to test performance. All analyses using these variables as between-group factors in analyses of composite cognitive variables were nonsignificant.

To determine whether DMD is associated with substantial deficits relative to the population norm, the percentage of children scoring below 1.5 standard deviations from the population mean was determined post hoc for each cognitive domain. The value of 1.5 standard deviations was chosen arbitrarily to be comparable to criteria often used in discrepancy analyses. The percentage of children with DMD whose scores fell below this cut-point was compared with the percentage of unaffected family members scoring below the same cut-point for each cognitive domain using $\chi^{2}$ analyses.

To examine the association between parent-reported adaptive skills and children's cognitive performance, post hoc correlational analyses were run on the different domain data.

For all analyses, alpha was set at the .05 level. For those analyses which also examined specific subtest differences, the Bonferroni correction was used (i.e., .05/number of specific subtests within each domain) to correct for multiple comparisons within each domain. Within each domain, individual $t$-values that were equal or less than the Bonferronicorrected alpha (e.g., $p<.025$ for a two-test domain) were considered to reflect significant between group differences.

\section{RESULTS}

\section{Adaptive Behavior}

According to parental report, children with DMD display significant delays in adaptive behavior skills. These delays are observed across domains when compared with their unaffected family members (see Table 1). Paired analyses indicate that children with DMD are delayed relative to familial controls in all four areas of functioning, including communication, daily living, socialization, and motor skills. In general, children with DMD were rated approximately 1 $S D$ lower than their controls across the scales with the biggest differences being observed on the communication and motor scales.

\section{Neuropsychological Test Data}

Children with DMD perform significantly more poorly than their unaffected family members in multiple areas of cognition (see Table 2). Paired analyses demonstrated that the boys with DMD performed more poorly than their controls on measures of receptive language, expressive language, visuo-spatial skills, fine-motor skills, attention, and memory. Thus, the profile appears to be one of global cognitive delay, with the probands scoring approximately one standard deviation below their familial controls.

A visual presentation of the performance of individual sibling pairs on one of the composite scores (e.g., expressive language) is presented in Figure 1. This figure demon-

Table 1. Comparison of adaptive functioning between probands and controls

\begin{tabular}{lcccccc}
\hline \hline & \multicolumn{2}{c}{ Probands } & & Controls & & \\
\cline { 2 - 2 } Vineland domain & Mean $(S D)$ & & Mean $(S D)$ & & \multirow{2}{*}{-value (df) } & Effect size \\
\hline Communication & $81.47(16.57)$ & & $107.26(15.37)$ & $4.90^{\mathrm{a}}(1,18)$ & $r=.76$ \\
Daily living skills & $78.68(19.18)$ & & $99.00(16.87)$ & $3.55^{\mathrm{a}}(1,18)$ & $r=.64$ \\
Socialization & $89.11(16.48)$ & & $105.79(15.52)$ & $3.90^{\mathrm{a}}(1,18)$ & $r=.68$ \\
Motor skills & $69.50(20.97)$ & & $94.25(14.67)$ & $3.40^{\mathrm{a}}(1,7)$ & $r=.79$ \\
\hline \hline
\end{tabular}

${ }^{\mathrm{a}} p<.05$. 
Table 2. Comparison of cognitive functioning between probands and controls

\begin{tabular}{|c|c|c|c|c|c|}
\hline & Probands & Controls & & & \\
\hline Cognitive domain & Mean $(S D)$ & Mean $(S D)$ & Pair diff & $t$-value (df) & Effect size \\
\hline Receptive language & $92.13(19.79)$ & $106.55(14.90)$ & -14.42 & $3.77^{\mathrm{a}}(1,18)$ & $r=.66$ \\
\hline PPVT-III & $98.15(20.24)$ & $106.05(12.41)$ & -7.90 & $2.48^{\mathrm{b}}(1,19)$ & \\
\hline CELF-P: Receptive & $86.74(21.18)$ & $107.05(19.05)$ & -20.32 & $3.85^{\mathrm{b}}(1,18)$ & \\
\hline Expressive language & $84.55(17.32)$ & $104.63(15.39)$ & -20.08 & $5.85^{\mathrm{a}}(1,18)$ & $r=.81$ \\
\hline EVT & $88.50(21.28)$ & $107.85(15.53)$ & -19.35 & $4.29^{\mathrm{b}}(1,19)$ & \\
\hline CELF-P: Expressive & $81.47(16.38)$ & $100.79(16.22)$ & -19.32 & $5.97^{\mathrm{b}}(1,18)$ & \\
\hline Visuo-spatial skills & $90.74(13.36)$ & $107.45(13.34)$ & -16.71 & $4.18^{\mathrm{a}}(1,18)$ & $r=.71$ \\
\hline Matching & $85.10(17.77)$ & $104.80(14.40)$ & -19.70 & $4.22^{\mathrm{b}}(1,19)$ & \\
\hline Picture completion & $96.05(19.26)$ & $109.74(16.11)$ & -13.68 & $2.41 \quad(1,18)$ & \\
\hline Fine-motor skills & $86.63(11.52)$ & $102.13(10.96)$ & -15.50 & $4.44^{\mathrm{a}}(1,19)$ & $r=.71$ \\
\hline Attention skills & $87.65(13.36)$ & $113.00(14.29)$ & -25.35 & $5.93^{\mathrm{a}}(1,16)$ & $r=.81$ \\
\hline Memory skills & $81.47(16.28)$ & $99.12(13.019)$ & -17.65 & $4.81^{\mathrm{a}}(1,16)$ & $r=.74$ \\
\hline
\end{tabular}

Note. Receptive language, Expressive language, and Visuo-spatial skills are multiple-test domains. Fine-motor skills, Attention skills, and Memory skills are single-test domains. PPVT-III = Peabody Picture Vocabulary Test, $3^{\text {rd }}$ edition; CELF-P = Clinical Evaluation of Language Fundamentals-Preschool Version; EVT = Expressive Vocabulary Test.

${ }^{\mathrm{a}} p<.05$.

${ }^{\mathrm{b}} p<.025$.

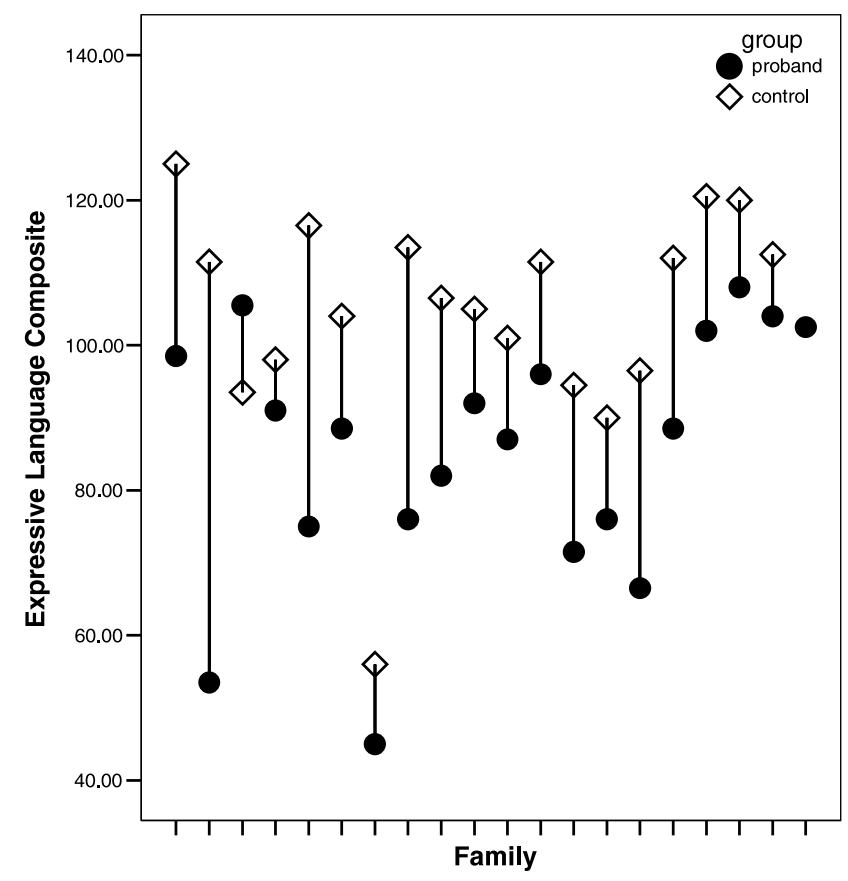

Fig. 1. Intra-pair differences in expressive language composite scores.

strates that, despite variability in their test scores, probands generally perform more poorly than their familial controls.

Based on the frequency of scores that were deficient relative to normative standards (defined here as 1.5 standard deviations below the normative age mean), cognitive deficits were more common among children with DMD as compared to unaffected family members (see Table 3 ). Moreover, results indicate that the likelihood of a child with DMD having deficits was greater in some cognitive domains than in others. Comparisons of scores that were $1.5 S D$ lower than the expected mean showed children with DMD differed from their controls in the expressive language, attention and memory domains, while the groups did not differ in the receptive language, fine-motor, and visual-spatial domains.

Results of the correlational analysis examining the association between parent-reported adaptive behaviors and child cognitive performance showed significant associations at the .05 level for many of the variables in both groups (see Table 4), as anticipated due to the known association between adaptive behavior and cognitive test score measure design (Sparrow et al., 1984). When more stringent criteria ( $\alpha$ of .001) were set, results indicated that for the DMD group, but not the controls, parents' adaptive ratings were associated with their child's performance. This finding likely reflects increased variability of performance across the probands. In addition, among the children with DMD, communication scores were strongly associated with cognitive outcomes whereas motor scores were not. This finding is intriguing and suggests that motor functioning may be less useful than communication skills as a marker of cognitive abilities in children with DMD.

\section{DISCUSSION}

The results of the current study indicate that young children with DMD exhibit impairments in multiple areas of cognitive and adaptive functioning, as confirmed both by parental report and neuropsychological test data. Parents report generalized deficits in adaptive functioning in their affected children. Children with DMD reportedly have difficulty in adaptive behaviors relating to language skills, motor skills, 
Table 3. Percentage of children who score more than $1.5 S D$ from the population mean

\begin{tabular}{llll}
\hline \hline Cognitive domain & $\begin{array}{c}\text { No. of } \\
\text { probands }(\%)\end{array}$ & $\begin{array}{c}\text { No. of } \\
\text { controls }(\%)\end{array}$ & $\begin{array}{l}\chi^{2} \text { with } \\
1 \mathrm{df}(\mathrm{N})\end{array}$ \\
\hline Receptive language & $3 / 20(15 \%)$ & $1 / 19(5.3 \%)$ & $1.00(39)$ \\
Expressive language & $7 / 20(35 \%)$ & $1 / 19(5.3 \%)$ & $5.28^{\mathrm{a}(39)}$ \\
Visuo-spatial skills & $3 / 19(15.8 \%)$ & $1 / 19(5.3 \%)$ & $1.12(38)$ \\
Fine-motor skills & $3 / 20(15 \%)$ & $0 / 20(0 \%)$ & $3.24(40)$ \\
Attention skills & $6 / 17(35.3 \%)$ & $0 / 17(0 \%)$ & $7.29^{\mathrm{a}}(34)$ \\
Memory skills & $6 / 17(35.3 \%)$ & $0 / 17(0 \%)$ & $7.29^{\mathrm{a}}(34)$ \\
\hline \hline
\end{tabular}

${ }^{\mathrm{a}} p<.05$.

personal care, and social skills. The children's skills were, on average, approximately one standard deviation below both normative expectations and familial controls.

Neuropsychological test data also showed generalized deficits across domains in young children with DMD. Specifically, when compared with familial controls of comparable age, young children with DMD performed significantly more poorly on tests of receptive and expressive language, visual-spatial skills, fine-motor skills, attention, and memory skills. Similar to the adaptive behavior findings, children with DMD scored approximately one standard deviation below their controls. In addition, more children with DMD had deficit scores in the expressive language, attention, and memory domains than their controls, suggesting that the DMD group was at a higher risk for clinically significant problems.

It is important to note, however, that despite significant between-group differences on all measures, children with DMD generally scored in the low-average to average ranges. Unaffected family members scored in the average to high- average ranges. As such, although group performance was shifted down, individual performance of some children with DMD might well have escaped attention had they not been compared with well-matched controls.

The results of the current study are in concordance with the previously published, albeit limited, data on cognitive deficits in young children with DMD. Smith et al. (1990) found generalized developmental delays, with the most severe impairments in language and motor skills. The delays reported in Smith's sample are more severe than those reported in the current study; sampling differences may be responsible for the discrepant findings. The current findings of generalized delay are also consistent with the case studies mentioned previously (Essex \& Roper, 2001; Kaplan et al., 1986; Mohamed et al., 2000), in which preschool children initially referred for language and behavioral delays were subsequently diagnosed with DMD.

The results of the current study are also consistent with prior cross-sectional studies which have found more severe language deficits in "younger" children with DMD, as com-

Table 4. Association of parent ratings of adaptive behavior with children's performance on neuropsychological tests

\begin{tabular}{|c|c|c|c|c|c|c|}
\hline \multirow{2}{*}{$\begin{array}{l}\text { Adaptive } \\
\text { behavior } \\
\text { domains }\end{array}$} & \multicolumn{6}{|c|}{ Neuropsychological domains } \\
\hline & $\begin{array}{l}\text { Receptive } \\
\text { language }\end{array}$ & $\begin{array}{c}\text { Expressive } \\
\text { language }\end{array}$ & Visuo-spatial & Fine-motor & Attention & Memory \\
\hline Communication & & & & & & \\
\hline Daily living skill & & & & & & \\
\hline Socialization & & & & & & \\
\hline Motor skills & & & $\mathrm{ns}$ & ns & & \\
\hline
\end{tabular}

Note. Scores for Receptive language, Expressive language, and Visuo-spatial skills were domain composites. ABS $=$ Adaptive Behavior Scales. Data presented are Pearson $r$ values. For each cell, values for DMD participants are above the diagonal and values for control participants are below the diagonal.

${ }^{\mathrm{a}} p<.05$.

${ }^{\mathrm{b}} p \leq .001$ 
pared to "older" children with DMD (Miller et al., 1985; Sollee et al., 1985), notwithstanding the fact that the "younger" children in previous samples (e.g., mean age 13.5 years for Miller et al., 1985, and mean age 7.7 years for Sollee et al., 1985) were considerably older than the young children in the current sample. The results of the current study also complement the cross-sectional findings of Cotton et al. (2005). According to a recent meta-analysis by Cotton and colleagues, young children with DMD (e.g., mean age 7 years, 5 months) exhibit significant verbal impairments. Furthermore, Cotton's cross-sectional data seem to indicate that verbal skills consistently improve with age in children and adolescents with DMD, as the oldest children outperformed those in all other age groups.

The findings of generalized deficits in young children with DMD (in our study and others) are at odds with the specificity of findings in older children and adolescents with this disorder. It appears that these deficits become more selective over time as the children mature. Interestingly, when the data are examined according to the percentage of children with deficit scores, the findings are more specific and similar to those reported in older children with DMD. That is, the children in the current sample did not have a significantly higher rate of deficits on tests of receptive language and visual-spatial skills, two areas that have been found to be relatively spared in older boys with DMD. Thus, even though scores from the boys with DMD were lower than their controls, they were nonetheless deficient only in areas previously shown to be impaired in DMD (e.g., verbal memory, expressive language, and attention).

Longitudinal data from children with early language impairments indicate that it is not unusual for language deficits to become less severe, or in fact, resolve completely over the course of the preschool years (Bishop \& Edmundson, 1987; Scarborough \& Dobrich, 1990; Silva, 1980). Some investigators (Aram \& Nation, 1975) have hypothesized that "the younger a child with a developmental language disorder, the more generalized is its effect, while as children become older, their language improves and the areas of deficiency become more specific" (p. 239). This hypothesis is consistent with Werner's (1948) characterization of cognitive development as "increasing differentiation and refinement of mental phenomena and functions and a progressive hierarchization" (p. 51). A longitudinal study, following young, preschool-aged children with DMD over time would be necessary to address the mechanism by which these generalized cognitive impairments resolve into specific language deficits. To investigate this issue, our lab is currently following children identified in this study and investigating their language and reading skills as they emerge.

One potential drawback of the current study is that it consisted of a sample of convenience. Given that there are currently no population-based studies which report upon the incidence of cognitive impairment in children with DMD, it is impossible at this time to determine whether the current sample over-represents cognitive impairments in this population. A second drawback to the current study-the small sample size-is of less concern because the strength of the effect sizes suggests that a lack of power was not at issue. Finally, the authors recognize that the lack of an appropriate IQ measure limits the interpretability of the current study. It is possible that differences in general intellectual functioning account for the multiple between group differences seen in these young children. A measure of IQ would aid in making such a determination.

The current study is particularly valuable for examining cognition in a proscribed age range (e.g., 3-6 years), allowing for a more fine-tuned analysis of language skills as they emerge. Other studies investigating cognition in DMD have used wide age ranges, which may mask subtle differences in the emergence of language. In addition, the current investigation extends prior findings by showing the functional impact in adaptive skills for these children. Finally, the use of familial controls allows the investigators to control for socio-economic variables, such as educational access, parenting style, social supports, and other environmental factors.

The cause of the cognitive deficits observed in children and adolescents with DMD is unknown. However, the deficits may be attributed to the lack of dystrophin, a protein product that is normally found in multiple tissues throughout the body. The genetic mutation responsible for DMD disrupts the production of dystrophin (Hoffman et al., 1987). Dystrophin is normally found in the neuromuscular junction of skeletal muscle fiber and ensures the structural integrity of the muscle (Blake et al., 2002). It is the absence of dystrophin in the skeletal muscle that causes the phenotypical presentation of progressive muscular weakness. However, dystrophin is also normally present in multiple tissues throughout the body, including the central nervous system, and in DMD its deficiency is not limited to skeletal muscle tissue (Lidov et al., 1990, 1993). Studies have documented the absence of dystrophin in the cerebral and cerebellar cortices of individuals diagnosed with DMD (Kim et al., 1995; Uchino et al., 1994a,b), providing a neural basis for the cognitive deficits in DMD. Development of a brain without dystrophin may lead to a brain that is "wired" differently from most, and may in turn lead to alterations in cognitive and behavioral development.

In summary, the current study examines adaptive behavior and cognitive deficits in a sample of children with a known genetic disorder. In contrast to the profile of specific verbal deficits reported in older children with DMD, young children with this disorder appear to exhibit generalized deficits in multiple areas of cognition and adaptive functioning. The current study helps elucidate genotypephenotype associations that are seen early in the disorder; however, it is not known how these generalized cognitive deficits evolve into more specific language impairments as children mature. It is hoped that future studies will help characterize the developmental trajectory in children with this disorder. The association of a specific cognitive profile with a genetic disorder is unique, and DMD offers the rare opportunity to examine such gene-cognition relationships. 


\section{ACKNOWLEDGMENTS}

This work was supported by grants from NINDS (R01 NS04791806A2) and the Muscular Dystrophy Association to V.J. Hinton.

\section{REFERENCES}

Adams, A.M. \& Gathercole, S.E. (2000). Limitations in working memory: Implications for language development. International Journal of Language \& Communication Disorders, 35, 95-116.

Adams, W. \& Sheslow, D. (1995). Wide Range Assessment of Visual Motor Abilities (WRAVMA). Wilmington, DE: Wide Range, Inc.

Anderson, S.W., Routh, D.K., \& Ionasescu, V.V. (1988). Serial position memory of boys with Duchenne muscular dystrophy. Developmental Medicine and Child Neurology, 30, 328-333.

Aram, D.M. \& Nation, J.E. (1975). Patterns of language behavior in children with developmental language disorders. Journal of Speech and Hearing Research, 18, 229-241.

Billard, C., Gillet, P., Barthez, M., Hommet, C., \& Bertrand, P. (1998). Reading ability and processing in Duchenne muscular dystrophy and spinal muscular atrophy. Developmental Medicine and Child Neurology, 40, 12-20.

Billard, C., Gillet, P., Signoret, J.L., Uicaut, E., Bertrand, P., Fardeau, M., Barthez-Carpentier, M.A., \& Santini, J.J. (1992). Cognitive functions in Duchenne muscular dystrophy: A reappraisal and comparison with spinal muscular atrophy. Neuromuscular Disorders, 2, 371-378.

Bishop, D.V. \& Edmundson, A. (1987). Language-impaired 4-yearolds: Distinguishing transient from persistent impairment. Journal of Speech and Hearing Disorders, 52, 156-173.

Blake, D.J., Weir, A., Newey, S.E., \& Davies, K.E. (2002). Function and genetics of dystrophin and dystrophin-related proteins in muscle. Physiological Review, 82, 291-329.

Cotton, S., Crowe, S.F., \& Voudouris, N. (1998). Neuropsychological profile of Duchenne muscular dystrophy. Child Neuropsychology, 4, 110-117.

Cotton, S., Voudouris, N.J., \& Greenwood, K.M. (2001). Intelligence and Duchenne muscular dystrophy: Full-scale, verbal, and performance intelligence quotients. Developmental Medicine and Child Neurology, 43, 497-501.

Cotton, S.M., Voudouris, N.J., \& Greenwood, K.M. (2005). Association between intellectual functioning and age in children and young adults with Duchenne muscular dystrophy: Further results from a meta-analysis. Developmental Medicine and Child Neurology, 47, 257-265.

Dorman, C., Hurley, A.D., \& D’Avignon, J. (1988). Language and learning disorders of older boys with Duchenne muscular dystrophy. Developmental Medicine and Child Neurology, 30, 316-327.

Dunn, L.M. \& Dunn, L.M. (1997). Examiner's manual for the PPVT-III Peabody Picture Vocabulary Test (3rd ed.). Circle Pines, MN: American Guidance Service.

Emery, A. \& Muntoni, F. (2003). Duchenne muscular dystrophy (3rd ed.). Oxford: Oxford University Press.

Essex, C. \& Roper, H. (2001). Late diagnosis of Duchenne's muscular dystrophy presenting as global developmental delay. British Medical Journal, 323, 37-38.

Gathercole, S.E., Hitch, G.J., Service, E., \& Martin, A.J. (1997). Phonological short-term memory and new word learning in children. Developmental Psychology, 33, 966-979.
Hendriksen, J.G. \& Vles, J.S. (2006). Are males with Duchenne muscular dystrophy at risk for reading disabilities? Pediatric Neurology, 34, 296-300.

Hinton, V.J., De Vivo, D.C., Fee, R., Goldstein, E., \& Stern, Y. (2004). Investigation of poor academic achievement in children with Duchenne muscular dystrophy. Learning Disabilities Research \& Practice, 19, 146-154.

Hinton, V.J., De Vivo, D.C., Nereo, N.E., Goldstein, E., \& Stern, Y. (2000). Poor verbal working memory across intellectual level in boys with Duchenne dystrophy. Neurology, 54, $2127-2132$.

Hinton, V.J., De Vivo, D.C., Nereo, N.E., Goldstein, E., \& Stern, Y. (2001). Selective deficits in verbal working memory associated with a known genetic etiology: The neuropsychological profile of Duchenne muscular dystrophy. Journal of the International Neuropsychological Society, 7, 45-54.

Hinton, V.J., Fee, R.J., Goldstein, E.M., \& De Vivo, D.C. (2007). Verbal and memory skills in males with Duchenne muscular dystrophy. Developmental Medicine and Child Neurology, 49, 123-128.

Hoffman, E.P., Brown, R.H., \& Kunkel, L.M. (1987). Dystrophin: The protein product of the Duchenne muscular dystrophy locus. Cell, 51, 919-928.

Kaplan, L.C., Osborne, P., \& Elias, E. (1986). The diagnosis of muscular dystrophy in patients referred for language delay. Journal of Child Psychology and Psychiatry, 27, 545-549.

Karagan, N.J., Richman, L.C., \& Sorensen, J.P. (1980). Analysis of verbal disability in Duchenne muscular dystrophy. Journal of Nervous and Mental Disease, 168, 419-423.

Kim, T.W., Wu, K., \& Black, I.B. (1995). Deficiency of brain synaptic dystrophin in human Duchenne muscular dystrophy. Annals of Neurology, 38, 446-449.

Korkman, M., Kirk, U., \& Kemp, S. (1997). NEPSY. San Antonio, TX: Harcourt Assessment, Inc.

Leibowitz, D. \& Dubowitz, V. (1981). Intellect and behavior in Duchenne muscular dystrophy. Developmental Medicine and Child Neurology, 23, 577-590.

Lidov, H.G., Byers, T.J., \& Kunkel, L.M. (1993). The distribution of dystrophin in the murine central nervous system: An immunocytochemical study. Neuroscience, 54, 167-187.

Lidov, H.G., Byers, T.J., Watkins, S.C., \& Kunkel, L.M. (1990). Localization of dystrophin to postsynaptic regions of central nervous system cortical neurons. Nature, 348, 725-728.

Miller, G., Tunnecliffe, M., \& Douglas, P.S. (1985). IQ, prognosis and Duchenne muscular dystrophy. Brain \& Development, 7 , $7-9$

Mohamed, K., Appleton, R., \& Nicolaides, P. (2000). Delayed diagnosis of Duchenne muscular dystrophy. European Journal of Paediatric Neurology, 4, 219-223.

Ogasawara, A. (1989). Downward shift in IQ in persons with Duchenne muscular dystrophy compared to those with spinal muscular atrophy. American Journal of Mental Retardation, 93, 544-547.

Scarborough, H.S. \& Dobrich, W. (1990). Development of children with early language delay. Journal of Speech and Hearing Research, 33, 70-83.

Silva, P.A. (1980). The prevalence, stability and significance of developmental language delay in preschool children. Developmental Medicine and Child Neurology, 22, 768-777.

Smith, R.A., Sibert, J.R., \& Harper, P.S. (1990). Early development of boys with Duchenne muscular dystrophy. Developmental Medicine and Child Neurology, 32, 519-527. 
Sollee, N.D., Latham, E.E., Kindlon, D.J., \& Bresnan, M.J. (1985). Neuropsychological impairment in Duchenne Muscular Dystrophy. Journal of Clinical and Experimental Neuropsychology, 7, 486-496.

Sparrow, S.S., Balla, D.A., \& Cicchetti, D.V. (1984). Vineland Adaptive Behavior Scales: Interview Edition/Survey Form Manual. Circle Pines, MN: American Guidance Service, Inc.

Uchino, M., Teramoto, H., Naoe, H., Miike, T., Yoshioka, K., \& Ando, M. (1994a). Dystrophin and dystrophin-related protein in the central nervous system of normal controls and Duchenne muscular dystrophy. Acta Neuropathologica, 87, 129-134.

Uchino, M., Teramoto, H., Naoe, H., Yoshioka, K., Miike, T., \& Ando, M. (1994b). Localisation and characterisation of dystrophin in the central nervous system of controls and patients with Duchenne muscular dystrophy. Journal of Neurology, Neurosurgery, and Psychiatry, 57, 426-429.

Wechsler, D. (1989). Wechsler Preschool and Primary Scale of Intelligence-Revised (WPPSI-R). San Antonio, TX: Harcourt Assessment, Inc.

Wechsler, D. (1991). Wechsler Intelligence Scale for ChildrenThird Edition (WISC-III). San Antonio, TX: Harcourt Assessment, Inc.
Werner, H. (1948). Comparative Psychology of Mental Development. New York: International Universities Press, Inc.

Whelan, T.B. (1987). Neuropsychological performance of children with Duchenne muscular dystrophy and spinal muscular atrophy. Developmental Medicine and Child Neurology, 29, 212-220.

Wicksell, R.K., Kihlgren, M., Melin, L., \& Eeg-Olofsson, O. (2004). Specific cognitive deficits are common in children with Duchenne muscular dystrophy. Developmental Medicine and Child Neurology, 46, 154-159.

Wiig, E.H., Secord, W., \& Semel, E. (1992). Clinical Evaluation of Language Fundamentals-Preschool (CELF-P) Examiner's Manual. San Antonio, TX: The Psychological Corporation.

Williams, K.T. (1997). Expressive Vocabulary Test (EVT). Circle Pines, MN: American Guidance Service.

Worden, D.K. \& Vignos, P.J. (1962). Intellectual function in childhood progressive muscular dystrophy. Pediatrics, 29, 968-977. 